Research Paper

\title{
Prognostic value of neutrophil-to-lymphocyte ratio, lactate dehydrogenase, D-dimer, and computed tomography score in patients with coronavirus disease 2019
}

\author{
Yu-Qing Caii,2, ${ }^{*}$, Xiao-Bin Zhang ${ }^{2,}{ }^{,}$, Hui-Qing Zeng ${ }^{1,2}$, Xiao-Jie Wei ${ }^{3}$, Lan $\mathrm{Hu}^{4}$, Zhen-Yu Zhang ${ }^{5}$, \\ Quan Ming 6 , Qiu-Ping Peng' ${ }^{6}$ Li-Da Chen ${ }^{7}$ \\ ${ }^{1}$ Department of Clinical Medicine, Fujian Medical University, Fujian, China \\ ${ }^{2}$ Department of Pulmonary and Critical Care Medicine, Zhongshan Hospital, Xiamen University, Teaching Hospital \\ of Fujian Medical University, Fujian, China \\ ${ }^{3}$ Department of Pulmonary and Critical Care Medicine, Third People's Hospital Affiliated to Fujian University of \\ Traditional Chinese Medicine, Fujian, China \\ ${ }^{4}$ Department of Gastroenterology, Optic Valley Division of Tongji Hospital, Tongji Medical College, Huazhong \\ University of Science and Technology, Wuhan, China \\ ${ }^{5}$ Department of Geriatrics, Zhongshan Hospital, Xiamen University, Teaching Hospital of Fujian Medical University, \\ Fujian, China \\ ${ }^{6}$ Yichang Third People's Hospital, Third People's Hospital Affiliated to Sanxia University, Hubei, China \\ ${ }^{7}$ Department of Pulmonary and Critical Care Medicine, Zhangzhou Affiliated Hospital of Fujian Medical University, \\ Fujian, China \\ *Equal contribution
}

Correspondence to: Hui-Qing Zeng; email: zhq20071212@xmu.edu.cn

Keywords: COVID-19, neutrophil-to-lymphocyte ratio, lactate dehydrogenase, D-dimer, CT score

Received: January 25, $2021 \quad$ Accepted: August 24, $2021 \quad$ Published: September 8, 2021

Copyright: (c) 2021 Cai et al. This is an open access article distributed under the terms of the Creative Commons Attribution License (CC BY 3.0), which permits unrestricted use, distribution, and reproduction in any medium, provided the original author and source are credited.

\section{ABSTRACT}

Background: This study aimed to explore the significance of neutrophil-to-lymphocyte ratio (NLR), lactate dehydrogenase (LDH), D-dimer, and CT score in evaluating the severity and prognosis of coronavirus disease 2019 (COVID-19).

Methods: Patients with laboratory-confirmed COVID-19 were retrospectively enrolled. The baseline data, laboratory findings, chest computed tomography (CT) results evaluated by CT score on admission, and clinical outcomes were collected and compared. Logistic regression was used to assess the independent relationship between the baseline level of the four indicators (NLR, LDH, D-dimer, and CT score) and the severity of COVID-19. Results: Among the 432 patients, 125 (28.94\%) and 307 (71.06\%) were placed in the severe and non-severe groups, respectively. As per the multivariate logistic regression, high levels of NLR and LDH were independent predictors of severe COVID-19 (OR=2.163; 95\% $\mathrm{Cl}=1.162-4.026 ; p=0.015$ for $\mathrm{NLR}>3.82 ; \mathrm{OR}=2.298 ; 95 \% \mathrm{Cl}=1.327$ 3.979; $p=0.003$ for $L D H>246 \mathrm{U} / \mathrm{L})$. Combined NLR $>3.82$ and $L D H>246 \mathrm{U} / \mathrm{L}$ increased the sensitivity of diagnosis in patients with severe disease (NLR>3.82 [50.40\%] vs. combined diagnosis [72.80\%]; $p=0.0007$; LDH>246 [59.2\%] vs. combined diagnosis [72.80\%]; $p<0.0001)$.

Conclusions: High levels of serum NLR and LDH have potential value in the early identification of patients with severe COVID-19. Moreover, the combination of LDH and NLR can improve the sensitivity of diagnosis. 


\section{INTRODUCTION}

Since December 2019, coronavirus disease-2019 (COVID-19), caused by a novel coronavirus (SARSCoV-2), has rapidly spread worldwide, causing a major public health issue [1]. COVID-19 is obviously a huge challenge for the global healthcare system [2], with the mortality of patients being related to the healthcare burden [3]. Therefore, a reasonable distribution of medical resources is particularly important. In turn, early identification of critical patients is crucial for the rational allocation of resources and the improvement of patient prognosis.

According to reports, hematological changes are more prominent in patients with severe COVID-19 than in patients with non-severe disease [4]. The neutrophil-tolymphocyte ratio (NLR), lactate dehydrogenase (LDH), and D-dimer are closely associated with the poor prognosis of COVID-19 [5, 6]. Without other clinical parameters, computed tomography (CT) evaluation is an independent prognostic factor in patients with COVID-19 [7]. However, there are few data comparing these four indicators. Therefore, in this study, we aimed to compare the prediction efficiency of NLR, LDH, Ddimer, and CT scores and evaluate the significance of the optimum cutoff. Subsequently, a combined diagnosis analysis was also performed to evaluate whether the combination of these indicators could improve diagnosis efficiency.

\section{RESULTS}

\section{Baseline, laboratory and imaging characteristics}

In this retrospective study, a total of 432 patients with COVID-19 were enrolled, including 202 (47\%) females and $230(53 \%)$ males with an average age of 52.88 years. Fever $(308,71.3 \%)$, cough $(270,62.5 \%)$, expectoration $(130,30.1 \%)$, and fatigue $(128,29.6 \%)$ were the most common symptoms. Hypertension (92, $21.3 \%$ ) was the most common comorbidity.

The patients have been divided into the severe $(125 / 432,28.94 \%)$ and non-severe $(307 / 432,71.06 \%)$ groups based on disease severity. In terms of the baseline characteristics, patients in the severe group had a more advanced average age than those in the nonsevere group $(59.60 \pm 16.65$ years vs. $50.14 \pm 16.26$ years, $p<0.0001)$. The severe group also had a higher incidence of comorbidities, such as hypertension $(p<0.0001)$, diabetes $(p<0.0001)$, and chronic obstructive pulmonary disease $(p=0.009)$. As for the clinical laboratory findings, lower levels of lymphocytes $(p<0.0001)$ and higher levels of white blood cells $(p=0.023)$, neutrophils $(p<0.0001)$, C- reaction proteins $(p<0.0001), \mathrm{LDH}(p<0.0001)$, D-dimer $(p<0.0001)$, and NLR $(p<0.0001)$ were detected in the severe group than in the non-severe group. Regarding the CT results, 96.0\% (120/125) of patients in the severe group had bilateral lung involvement, 32\% (40/125) had consolidation, and 3.2\% (4/125) had pleural effusion. A significant difference in terms of CT score was also observed between the two groups (6 [49] for the severe group vs. 6 [4-7] for the non-severe group, $p<0.0001$ ) (Table 1).

\section{Predictive value of NLR, LDH, D-dimer, and CT score}

As shown in Table 1, NLR, LDH, D-dimer, and CT scores were significantly higher in the severe group than in the non-severe group. Based on the receiver operating characteristic (ROC) curve, the area under the curve (AUC) was 0.716 for NLR, 0.740 for $\mathrm{LDH}, 0.650$ for D-dimer, and 0.612 for CT score, indicating a certain diagnostic value for the severity of disease (Figure 1 and Table 2). In addition, the optimum cutoff values from the ROC were $3.82,246 \mathrm{U} / \mathrm{L}, 0.83 \mu \mathrm{g} / \mathrm{mL}$, and 7 for NLR, LDH, D-dimer, and CT score, respectively (Table 2).

We assumed that when the levels of NLR, LDH, Ddimer, and CT score on admission exceeded the optimum cutoff, the patients were prone to develop severe or critical disease types. Patients were then divided into different subgroups according to the optimum cutoff.

As Table 3 shows, $25.9 \%$ (112/432), 31.9\% (138/432), $22.2 \%(96 / 432)$, and $25 \%(108 / 432)$ of patients had high levels of NLR, LDH, D-dimer, and CT score on admission, respectively. After grouping, the distribution of baseline NLR [63/125 (50.4\%) vs. 49/307 (16\%); $p<0.0001]$, LDH [74/125 (59.2\%) vs. 64/307 (20.8\%); $p<0.0001]$, D-dimer [47/125 (37.6\%) vs. 49/307 (16\%); $p<0.0001]$, and CT score [46/125 (36.8\%) vs. 62/307 $(20.2 \%) ; p<0.0001]$ over the optimum cutoff in the two groups were significant (Table 3 ).

Univariate analysis indicated that high levels of NLR, LDH, D-dimer, and CT score were positively correlated with disease severity $(\mathrm{OR}=5.350 ; 95 \% \mathrm{CI}=3.361-8.518$; $p<0.0001$ for $\mathrm{NLR}$; $\mathrm{OR}=5.509 ; 95 \% \mathrm{CI}=3.511-8.646$; $p<0.0001$ for $\mathrm{LDH}$; $\mathrm{OR}=3.173 ; 95 \% \mathrm{CI}=1.976-5.094$; $p<0.0001$ for $\mathrm{D}$-dimer; $\mathrm{OR}=2.301 ; 95 \% \mathrm{CI}=1.455$ 3.638; $p<0.0001$ for CT score). After adjusting for other statistically significant indices, the predictive value of NLR>3.82, LDH>246 U/L persisted $(\mathrm{OR}=2.163 ; 95 \%$ $\mathrm{CI}=1.162-4.026 ; p=0.015$ for $\mathrm{NLR} ; \mathrm{OR}=2.298 ; 95 \%$ $\mathrm{CI}=1.327-3.979 ; p=0.003$ for $\mathrm{LDH})$. By contrast, the relationship among D-dimer $>0.83 \mu \mathrm{g} / \mathrm{mL}$, CT score $>7$, 
Table 1. Baseline, laboratory and imaging characteristics.

\begin{tabular}{|c|c|c|c|c|}
\hline Variable & Total $(n=432)$ & Severe group $(n=125)$ & Non-severe group $(n=307)$ & $p$ value \\
\hline Age(years) & $52.88 \pm 16.91$ & $59.60 \pm 16.65$ & $50.14 \pm 16.26$ & $<0.0001$ \\
\hline Gender & & & & 0.072 \\
\hline Female-n $(\%)$ & 202(47) & $50(40)$ & $152(49.5)$ & \\
\hline Male-n $(\%)$ & $230(53)$ & $75(60)$ & $155(50.5)$ & \\
\hline \multicolumn{5}{|l|}{ Clinical symptom-n (\%) } \\
\hline Fever & 308(71.3) & $94(75.2)$ & $214(69.7)$ & 0.252 \\
\hline Fatigue & $128(29.6)$ & $46(36.8)$ & $82(26.7)$ & 0.037 \\
\hline Dyspnea & $35(8.1)$ & $20(16)$ & $15(4.9)$ & $<0.0001$ \\
\hline Pharyngalgia & $34(7.9)$ & $12(9.6)$ & $22(7.2)$ & 0.394 \\
\hline Cough & $270(62.5)$ & $84(67.2)$ & $186(60.6)$ & 0.198 \\
\hline Chest tightness & $47(10.9)$ & $22(17.6)$ & $25(8.1)$ & 0.004 \\
\hline Diarrhea & $20(4.6)$ & $6(4.8)$ & $14(4.6)$ & 0.914 \\
\hline Myalgia & $46(10.6)$ & $19(15.2)$ & $27(8.8)$ & 0.05 \\
\hline Expectoration & $130(30.1)$ & $35(28)$ & $95(30.9)$ & 0.545 \\
\hline Headache & $19(4.4)$ & $6(4.8)$ & $13(4.2)$ & 0.795 \\
\hline Poor appetite & $53(12.3)$ & $19(15.2)$ & $34(11.1)$ & 0.236 \\
\hline \multicolumn{5}{|l|}{ Comorbidities-n (\%) } \\
\hline Hypertension & $92(21.3)$ & $53(42.4)$ & $39(12.7)$ & $<0.0001$ \\
\hline Diabetes & $56(13)$ & $31(24.8)$ & $25(8.1)$ & $<0.0001$ \\
\hline COPD & $25(5.8)$ & $13(10.4)$ & $12(3.9)$ & 0.009 \\
\hline Renal insufficiency & $9(2.1)$ & $8(6.4)$ & $1(0.3)$ & $<0.0001$ \\
\hline Cardiac insufficiency & $8(1.9)$ & $7(5.6)$ & $1(0.3)$ & 0.01 \\
\hline Hepatic insufficiency & $30(6.9)$ & $16(12.8)$ & $14(4.6)$ & 0.002 \\
\hline Anemia & $13(3.0)$ & $7(5.6)$ & $6(2.0)$ & 0.089 \\
\hline \multicolumn{5}{|l|}{ Clinical laboratory } \\
\hline White blood cell- $10^{\wedge} 9 / \mathrm{L}$ & $5.25 \pm 2.52$ & $5.76 \pm 3.19$ & $5.04 \pm 2.16$ & 0.023 \\
\hline Lymphocyte- $10^{\wedge 9} / \mathrm{L}$ & $1.28 \pm 0.62$ & $1.04 \pm 0.70$ & $1.37 \pm 0.55$ & $<0.0001$ \\
\hline Neutrophil-10^^/L (IQR) & $2.98(2.11-4.18)$ & $3.41(2.32-5.50)$ & $2.82(2.08-3.77)$ & $<0.0001$ \\
\hline CRP-mg/L(IQR) & $22.32(9.15-37.7)$ & $45.2(14.85-55.8)$ & $22.32(7.7-22.32)$ & $<0.0001$ \\
\hline Platelet- $10^{\wedge} 9 / \mathrm{L}$ & $173.02 \pm 80.88$ & $163.03 \pm 83.74$ & $177.08 \pm 79.47$ & 0.102 \\
\hline D-dimer- $\mu \mathrm{g} / \mathrm{ml}$ (IQR) & $0.55(0.44-0.82)$ & $0.62(0.50-1.42)$ & $0.52(0.42-0.68)$ & $<0.0001$ \\
\hline LDH-U/L(IQR) & $210(170-267.75)$ & $265(207.5-356)$ & $196(162-235)$ & $<0.0001$ \\
\hline NLR (IQR) & $2.33(1.51-3.94)$ & $3.84(2.06-7.13)$ & $2.03(1.41-3.25)$ & $<0.0001$ \\
\hline \multicolumn{5}{|l|}{ CT manifestations } \\
\hline CT score (IQR) & $6(4-7.75)$ & $6(4-9)$ & $6(4-7)$ & $<0.0001$ \\
\hline Bilateral lung involved-n (\%) & $359(83.1)$ & $120(96)$ & $239(77.9)$ & $<0.0001$ \\
\hline Ground glass opacity-n (\%) & $426(98.6)$ & 124(99.2) & 302(98.4) & 0.830 \\
\hline Consolidation-n (\%) & $96(22.2)$ & $40(32)$ & $56(18.2)$ & 0.002 \\
\hline Pleural effusion-n (\%) & $5(1.2)$ & $4(3.2)$ & $1(0.3)$ & 0.042 \\
\hline Pleural thickening-n (\%) & $5(1.2)$ & $1(0.8)$ & $4(1.3)$ & 1.000 \\
\hline
\end{tabular}

Abbreviation: COPD, chronic obstructive pulmonary disease; CRP, C-reactive protein; NLR, neutrophil - to- lymphocyte ratio; $\mathrm{LDH}$, lactate dehydrogenase.

and disease severity weakened (OR=1.209; 95\% $\mathrm{CI}=0.626-2.334 ; p=0.571$ for $\mathrm{D}$-dimer; $\mathrm{OR}=1.519 ; 95 \%$ $\mathrm{CI}=0.71-3.247 ; p=0.281$ for $\mathrm{CT}$ score). In addition, fatigue $(\mathrm{OR}=1.978 ; 95 \% \quad \mathrm{CI}=1.127-3.473 ; p=0.018)$, chest tightness $(\mathrm{OR}=2.265 ; 95 \% \quad \mathrm{CI}=1.011-5.074$; $p=0.047)$, hypertension $(\mathrm{OR}=2.534,95 \% \mathrm{CI}=1.259$ $5.099 ; p=0.009)$, C-reactive protein $(\mathrm{OR}=1.013 ; 95 \% \mathrm{CI}=$ 1.003-1.023; $p=0.011$ ), and bilateral lung involvement $(\mathrm{OR}=3.890 ; 95 \% \mathrm{CI}=1.356-11.154 ; p=0.011)$ were still positively correlated with disease severity (Table 4 ). 


\section{Evaluation of the multi-parameter model}

According to logistic regression, NLR>3.82 and $\mathrm{LDH}>246 \mathrm{U} / \mathrm{L}$ were statistically significant risk factors (Table 4). As shown in Table 2, the sensitivity of NLR>3.82 and LDH>246 U/L in predicting the severity of COVID-19 were $50.40 \%$ and $59.20 \%$, respectively. Further evaluation was performed to judge whether the combined diagnosis model of the two indices can improve prediction sensitivity.

Table 5 indicates that the combined diagnosis of NLR>3.82 and LDH>246 U/L could increase the sensitivity of predicting disease severity [NLR $>3.82$ $(50.40 \%)$ vs. combined diagnosis model $(72.80 \%) ; p$ $=0.0007 ; \mathrm{LDH}>246(59.2 \%)$ vs. combined diagnosis model $(72.80 \%) ; p<0.0001]$.

\section{DISCUSSION}

A total of 432 patients with COVID-19 were included in this retrospective study. In the univariate analysis, we found that high levels of NLR, LDH, D-dimer, and CT score were significantly correlated with COVID-19 severity. After adjusting for other statistically significant indices, the predictive value of NLR $>3.82$ and $\mathrm{LDH}>246 \mathrm{U} / \mathrm{L}$ persisted. This indicates that when NLR exceeded the cutoff point, the risk of serious disease increased by 2.163 times. Moreover, the risk of LDH over the optimum cutoff increased by 2.298 times. By contrast, the value of D-dimer $>0.83 \mu \mathrm{g} / \mathrm{mL}$ and CT score $>7$ in predicting disease severity was weak and these indices could therefore not be recommended as independent predictors. In addition, the risk of severity was closely related to fatigue, chest tightness,
A

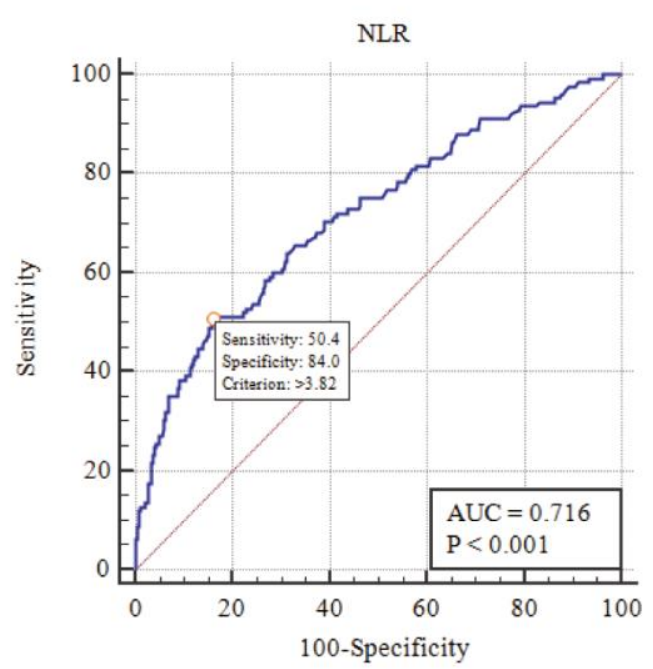

C

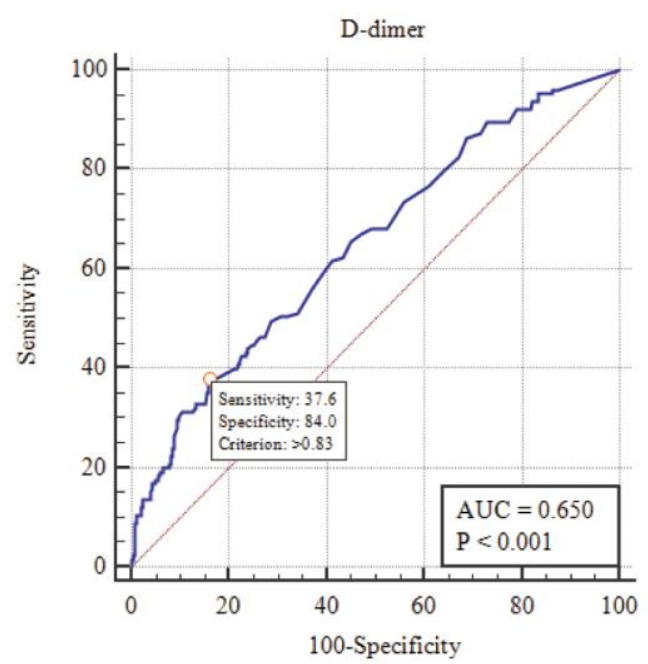

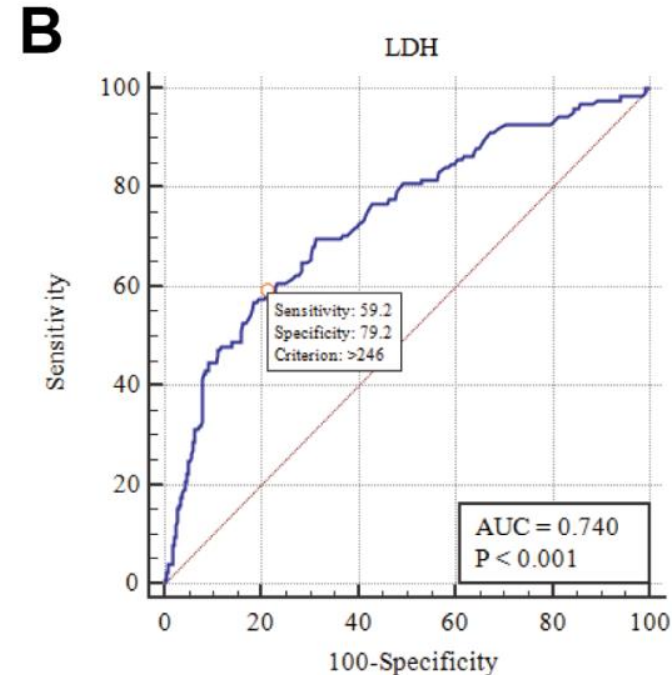

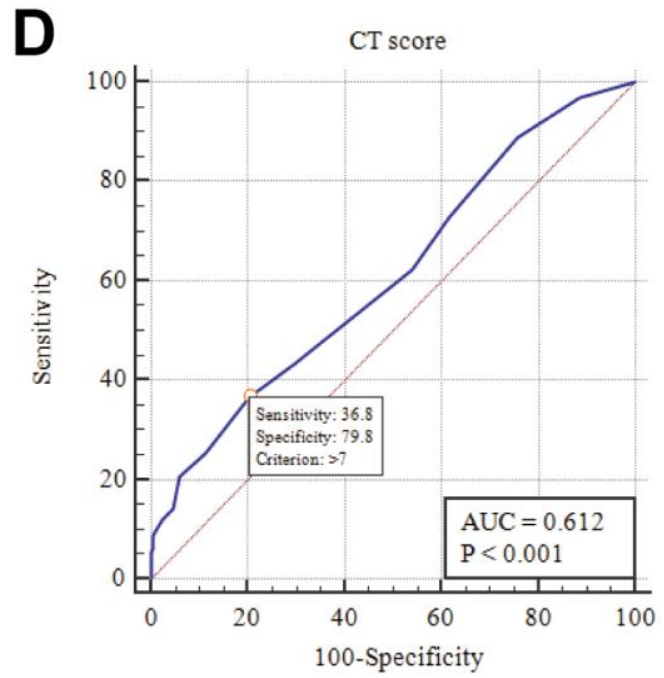

Figure 1. ROC analysis of NLR, LDH, D-dimer and CT score in disease risk prediction (A) NLR; (B) LDH; (C) D-dimer; (D) CT score. 
Table 2. Area under ROC curve and optimum cutoff.

\begin{tabular}{|c|c|c|c|c|c|c|c|c|}
\hline \multirow{3}{*}{ Variables } & \multicolumn{8}{|c|}{ Assessment of validity } \\
\hline & \multirow{2}{*}{$\mathbf{A U C}$} & \multirow{2}{*}{ Optimum cutoff } & \multirow{2}{*}{ Sensitivity } & \multirow{2}{*}{ Specificity } & \multicolumn{2}{|c|}{ Predictive value } & \multicolumn{2}{|c|}{ Likelihood ratio } \\
\hline & & & & & Positive & Negative & Positive & Negative \\
\hline NLR & 0.716 & 3.82 & $50.4 \%$ & $84.04 \%$ & $56.3 \%$ & $80.6 \%$ & 3.16 & 0.59 \\
\hline $\mathrm{LDH}(\mathrm{U} / \mathrm{L})$ & 0.740 & 246 & $59.2 \%$ & $79.15 \%$ & $53.6 \%$ & $82.7 \%$ & 2.84 & 0.52 \\
\hline $\mathrm{D}-\operatorname{dimer}(\mu \mathrm{g} / \mathrm{ml})$ & 0.650 & 0.83 & $37.6 \%$ & $84.04 \%$ & $49 \%$ & $76.8 \%$ & 2.36 & 0.74 \\
\hline CT-score & 0.612 & 7 & $36.8 \%$ & $79.8 \%$ & $42.6 \%$ & $75.6 \%$ & 1.82 & 0.79 \\
\hline
\end{tabular}

Abbreviation: ROC, receiver operator characteristic curve; NLR, neutrophil-to-lymphocyte ratio; LDH, lactate dehydrogenase.

Table 3. Baseline after grouping.

\begin{tabular}{|c|c|c|c|c|}
\hline & Total $n=432$ & Severe group $n=125$ & Non -severe group $n=307$ & $p$ value \\
\hline \multicolumn{5}{|l|}{ NLR } \\
\hline$>3.82$ & $112(25.9 \%)$ & $63(50.4 \%)$ & $49(16 \%)$ & \multirow{2}{*}{$p<0.0001$} \\
\hline$\leq 3.82$ & $320(74.1 \%)$ & $62(49.6 \%)$ & $258(84 \%)$ & \\
\hline \multicolumn{5}{|c|}{$\mathrm{LDH}(\mathrm{U} / \mathrm{L})$} \\
\hline$>246$ & $138(31.9 \%)$ & $74(59.2 \%)$ & $64(20.8 \%)$ & \multirow{2}{*}{$p<0.0001$} \\
\hline$\leq 246$ & $294(68.1 \%)$ & $51(40.8 \%)$ & $243(79.2 \%)$ & \\
\hline \multicolumn{5}{|c|}{$\mathrm{D}-\operatorname{dimer}(\mu \mathrm{g} / \mathrm{ml})$} \\
\hline$>0.83$ & $96(22.2 \%)$ & $47(37.6 \%)$ & $49(16 \%)$ & \multirow{2}{*}{$p<0.0001$} \\
\hline$\leq 0.83$ & $336(77.8 \%)$ & $78(62.4 \%)$ & $258(84 \%)$ & \\
\hline \multicolumn{5}{|c|}{ CT score } \\
\hline$>7$ & $108(25 \%)$ & $46(36.8 \%)$ & $62(20.2 \%)$ & \multirow{2}{*}{$p<0.0001$} \\
\hline$\leq 7$ & $324(75 \%)$ & $79(63.2 \%)$ & $245(79.8 \%)$ & \\
\hline
\end{tabular}

Abbreviation: ROC, receiver operator characteristic curve; NLR, neutrophil-to-lymphocyte ratio; LDH, lactate dehydrogenase.

Table 4. The univariate and multivariable logistic regression.

\begin{tabular}{lcccc}
\hline Variables & Unadjusted odds ratio $(95 \%$ CI $)$ & $\boldsymbol{p}$ value & Adjusted odds ratio (95\%CI) & $\boldsymbol{p}$ value \\
\hline NLR & $5.350(3.361-8.518)$ & $<0.0001$ & $2.163(1.162-4.026)$ & 0.015 \\
LDH(U/L) & $5.509(3.511-8.646)$ & $<0.0001$ & $2.298(1.327-3.979)$ & 0.003 \\
D-dimer $\mu \mathrm{g} / \mathrm{ml})$ & $3.173(1.976-5.094)$ & $<0.0001$ & $1.209(0.626-2.334)$ & 0.571 \\
CT score & $2.301(1.455-3.638)$ & $<0.0001$ & $1.519(0.71-3.247)$ & 0.281 \\
Age & $1.036(1.022-1.050)$ & $<0.0001$ & $0.994(0.975-1.014)$ & 0.561 \\
Fatigue & $1.598(1.026-2.488)$ & 0.038 & $1.978(1.127-3.473)$ & 0.018 \\
Dyspnea & $3.708(1.831-7.509)$ & $<0.0001$ & $1.348(0.507-3.585)$ & 0.55 \\
Chest tightness & $2.409(1.302-4.460)$ & 0.005 & $2.265(1.011-5.074)$ & 0.047 \\
Hypertension & $5.058(3.103-8.245)$ & $<0.0001$ & $2.534(1.259-5.099)$ & 0.009 \\
Diabetes & $3.720(2.091-6.619)$ & $<0.0001$ & $1.304(0.597-2.848)$ & 0.506 \\
COPD & $2.853(1.264-6.441)$ & 0.012 & $1.019(0.314-3.303)$ & 0.975 \\
Renal insufficiency & $20.923(2.589-169.118)$ & 0.004 & $4.788(0.449-51.025)$ & 0.195 \\
Cardiac insufficiency & $18.153(2.210-149.133)$ & 0.007 & $2.245(0.135-37.251)$ & 0.573 \\
Hepatic insufficiency & $3.072(1.451-6.505)$ & 0.003 & $2.209(0.842-5.792)$ & 0.107 \\
CRP (mg/L) & $1.025(1.017-1.033)$ & $<0.0001$ & $1.013(1.003-1.023)$ & 0.011 \\
Bilateral lung involved & $6.828(2.683-17.381)$ & $<0.0001$ & $3.890(1.356-11.154)$ & 0.011 \\
Consolidation & $2.109(1.312-3.390)$ & 0.002 & $1.303(0.6-2.829)$ & 0.504 \\
Pleural effusion & $10.116(1.119-91.421)$ & 0.039 & $5.097(0.409-63.513)$ & 0.206 \\
\hline
\end{tabular}

Abbreviation: NLR, neutrophil - to- lymphocyte ratio; LDH, lactate dehydrogenase; COPD, chronic obstructive pulmonary disease; CRP, C-reactive protein. 
Table 5. Comparison of univariate and combined diagnosis model.

\begin{tabular}{lccc}
\hline Variables & Sensitivity & Specificity & $\boldsymbol{p}$ value \\
\hline NLR $>3.82$ & $50.40 \%$ & $84.04 \%$ & $0.0007^{1}$ \\
LDH $>246 \mathrm{U} / \mathrm{L}$ & $59.20 \%$ & $79.15 \%$ & $<0.0001^{2}$ \\
Combined diagnosis model & $72.80 \%$ & $69.71 \%$ & \\
\hline
\end{tabular}

Abbreviation: NLR, neutrophil - to- lymphocyte ratio; $L D H$, lactate dehydrogenase; combined diagnosis model, NLR>3.82 and $\mathrm{LDH}>246 \mathrm{U} / \mathrm{L} ; 0.0007^{1}, p$ value between NLR>3.82 and combined diagnosis model; $<0.0001^{2}, p$ value between $\mathrm{LDH}>246 \mathrm{U} / \mathrm{L}$ and combined diagnosis model.

hypertension, and C-reactive protein. Furthermore, combining NLR>3.82 and LDH>246 U/L can improve the sensitivity of disease risk prediction.

Immune dysfunction plays an important role in the severity of COVID-19 [8]. Recent studies have elucidated that neutropenia and lower lymphopenia can be observed in patients with severe COVID-19 $[9,10]$. The NLR simultaneously considers the lymphocytes and neutrophils, and several studies have shown the predictive value of NLR in distinguishing patients with severe and critical COVID-19. In a study of the dynamic changes in lymphocyte subsets and cytokine profiles in patients with COVID-19, NLR was found to be a prognostic factor for the early identification of severe cases [11]. A cohort of patients with COVID-19 also proved that, after adjustment for confounding factors, the risk of in-hospital mortality increased by $8 \%$ for each unit increase in NLR [12]. Another study conducted by Yang et al. [5] in 93 patients with COVID-19 demonstrated that NLR can be used as an independent indicator for poor clinical outcome, and that the largest AUC for NLR was 0.841, with $63.6 \%$ specificity and $88 \%$ sensitivity. However, the outcome requires further evaluation because of limited sample diversity. The predictive value of NLR in the present study was consistent with the abovementioned studies. Moreover, the sample size and diversity in the present study were improved by collecting data from two clinical centers, which strengthens the reliability of our conclusions. We found that the optimum cutoff for NLR was 3.82, and the AUC was 0.716. Moreover, the sensitivity and specificity of NLR>3.82 were $50.40 \%$ and $84.04 \%$, respectively. Moreover, as per the multivariate logistic regression, NLR $>3.82$ can be used as an independent predictor for disease risk $(\mathrm{OR}=2.163$; 95\% $\mathrm{CI}=1.162-4.026 ; p=0.015$ ).

Elevation of LDH is one of the most common laboratory abnormalities in patients with COVID-19. Acute lung injury is highly associated with LDH [13]. A systematic literature review and meta-analysis showed that $\mathrm{LDH}$ levels $>245 \mathrm{U} / \mathrm{L}$ can predict the progression of COVID-19 [6]. In a study of the risk factors for death in cancer patients with COVID-19, elevated LDH levels were closely related to increased mortality [14]. Furthermore, in another retrospective analysis of 120 patients with COVID-19, the patients with severe disease had higher LDH levels than patients with mild disease (mean 200.8 U/L for mild vs. mean $342.8 \mathrm{U} / \mathrm{L}$ for severe) [15]. The predictive value of $\mathrm{LDH}$ was further confirmed in our study. Our ROC analysis showed that the AUC for LDH was 0.74, and that the optimum cutoff was $246 \mathrm{U} / \mathrm{L}$. The sensitivity and specificity were $59.2 \%$ and $79.15 \%$, respectively. Logistic regression indicated that the risk of serious disease increased by 2.298 times when LDH was above the optimum cutoff $(\mathrm{OR}=2.298 ; 95 \% \mathrm{CI}=1.327-3.979$; $p=0.003)$. In addition, the sensitivity of disease risk prediction can be improved by combining LDH $>246$ $\mathrm{U} / \mathrm{L}$ with NLR>3.82. (NLR>3.82 [50.40\%] vs. combined diagnosis model [72.80\%]; $p=0.0007$; LDH>246 [59.2\%] vs. combined diagnosis model [72.80\%]; $p<0.0001)$. However, the specificity was decreased (NLR $>3.82[84.04 \%]$ vs. combined diagnosis model [69.71\%]; $p=0.0007 ; \mathrm{LDH}>246$ [79.15\%] vs. combined diagnosis model [69.71\%]; $p<0.0001)$.

Furthermore, the sensitivity, specificity, and AUC for NLR and LDH were not sufficiently high. Due to the different admission times of patients with COVID-19 and the acute aggravation of some patients after admission, the value of admission indicators may have been underestimated. However, compared with other studies [5, 11, 16], the sample size and diversity of patients with COVID-19 have increased the reliability of the results in this study. More importantly, the optimum cutoff can indicate the risk of acute aggravation in patients with COVID-19 in the present study. Furthermore, our study provides more evidence for the establishment of a multiparameter diagnosis model. The combination of indicators increases the 
possibility of disease progression. And the role of primary screening in emergency needs to be further confirmed.

Coagulation disorders are more common in patients with severe disease than in patients with mild disease [17, 18]. A study conducted by Zhang et al. [19] showed that a D-dimer level $\geq 2.0 \mu \mathrm{g} / \mathrm{mL}$ (four-fold increase) could effectively predict the mortality of patients with COVID-19. In our study, after balancing the confounding factors, the logistic regression showed that D-dimer $>0.83 \mu \mathrm{g} / \mathrm{mL}$ could not be used as an independent predictor of disease risk $(\mathrm{OR}=1.209$; $95 \%$ $\mathrm{CI}=0.626-2.334 ; p=0.571)$. In a dynamic study of hematological parameters in patients with COVID-19, the D-dimer level was higher in the severe group than in the non-severe group on days 1,7 , and $14(p<0.05)$ [20]. This suggests that due to different admission times, the ability of D-dimer to predict disease risk may be weakened. In addition to the prognostic value of D-dimer in patients with COVID-19, the predictive value of $\mathrm{D}$-dimer might be affected by other factors, such as hormone therapy and antibiotic therapy. Because the baseline level of D-dimer varies greatly in patients, the value of D-dimer dynamic monitoring may be higher in patients with COVID-19 [21]. Nevertheless, further research is required to evaluate the significance of D-dimer in evaluating the severity of COVID-19.

Patients with COVID-19 have lung involvements with imaging changes $[22,23]$. In different stages of the disease, the CT manifestations are different, which are important for the diagnosis and staging of patients [24]. Using the same semi-quantitative scoring system, a multi-center paired cohort study conducted by Liu et al. [25] showed that CT changes are obvious during the acute exacerbation of COVID-19, accompanied by an increase in CT score. This indicates that an elevated CT score may predict a poor outcome. Another retrospective single-center study indicated that the CT score had a high diagnostic value in patients with severe COVID-19. ROC analysis showed that the AUC for the CT score was 0.918. The optimum cutoff CT score was 7.5. The sensitivity and specificity were $82.6 \%$ and $100 \%$, respectively [8]. However, the study only analyzed imaging without combining it with clinical data. Significant differences in the number of patients between the severe-critical and non-severe groups also affected the accuracy of the results. In the present study, after combining the clinical data, the CT score cannot be used as an independent predictor of disease risk $(\mathrm{OR}=1.519$; 95\% CI=0.71-3.247; $p=0.281$ ). A study by Zhang B et al. [26] demonstrated that the severity of lung abnormalities evaluated by CT score might be associated with laboratory parameters. Therefore, due to the correlation between CT score and laboratory parameters, the ability to independently predict the disease risk from CT scores may be attenuated. Additional investigations are warranted to assess whether CT score can be an independent predictor of disease risk.

This study has some limitations. First, owing to the different disease severities among the patients, as well as the different medical resources available, the time from onset to admission might not be representative, which could have affected the level of the four parameters considered on admission. Moreover, the representativeness of the $\mathrm{CT}$ score and D-dimer may have also been affected by the different admission times. Second, other clinical data and test results were not included in the analysis, which may have caused bias, weakening the reliability of the results. Third, it should be noted that the CT score was a subjective semi-quantitative evaluation method, to a certain degree. In the future research, it is necessary to conduct dynamic research on indicators and combine more indicators to meet different clinical needs.

\section{CONCLUSIONS}

As independent factors, the serum levels of NLR and LDH were significantly correlated with COVID-19 severity. Therefore, we recommend NLR and LDH as predictors for evaluating the severity of COVID-19.

\section{MATERIALS AND METHODS}

\section{Study design and participants}

From January 20, 2020, to March 30, 2020, a total of 432 patients confirmed COVID-19 by the laboratory in designated treatment hospitals (Optic Valley division of Tongji Hospital, Tongji Medical College, Huazhong University of Science and Technology, Wuhan and Yichang Third People's Hospital, Hubei Province) were enrolled. The patients were divided into 2 groups based on the seventh edition of the New Coronavirus Pneumonia Diagnosis and Treatment Program published by the Chinese National Health Commission [27]: the mild and moderate types were classified as non-severe group and the severe and critical were included into severe group. The disease is classified as severe if one of the following items is met: 1) shortness of breath, respiratory rate $\geq 30$ beats per min; 2) the oxygen saturation $\leq 93 \%$ in a resting state; 3) arterial partial pressure of oxygen $\left(\mathrm{PaO}_{2}\right)$ / concentration of oxygen $\left(\mathrm{FiO}_{2}\right) \leq 300 \mathrm{mmHg}(1 \mathrm{mmHg}$ $=0.133 \mathrm{kPa})$; 4) pulmonary images show that the lesions progressed more than $50 \%$ within $24-48 \mathrm{~h}$. The critical should meet one of the following conditions: 1) respiratory failure and need mechanical ventilation; 2 ) 
shock; and 3) other organ failures need ICU monitoring and treatment.

\section{Date collection}

The data of patients' demographic characteristics, comorbidities, laboratory findings, chest computed tomography (CT) results, and clinical outcomes were extracted from electronic medical records. The BC 3000 auto hematology analyzer (Mindray Medical International, Inc., Shenzhen, China) was used for routine blood tests of hospitalized patients. Biochemical and inflammatory markers were obtained on a Beckman Coulter AU5800 (Beckman Coulter Co, Brea, CA, USA). CT image acquisition and scoring A thoracic CT scan was performed before or after 2 days of admission in all patients. According to the extent of involvement of each lobe, each lobe was scored as $0(0 \%), 1$ (1$25 \%), 2(26-50 \%), 3(51-75 \%)$, or $4(76-100 \%)$. The total severity score (TSS) is the cumulative score of five lobes (score range $0-20)[8,28]$. In order to ensure the accuracy of the data, all data were checked by two physicians, respectively.

\section{Statistical analysis}

According to the different data distribution, continuous variables were described as mean \pm standard or median (Inter-quartile range, IQR), and groups were compared by student's t-test or Mann-Whitney U test based on the data distribution. Categorical variables were presented as $\mathrm{n}(\%)$ and analyzed by Pearson's chi-square. Receiver operator characteristic (ROC) was used to evaluate the efficacy of NLR, LDH, D-dimer and CT score and get the optimum cutoff. Logistic regression was used to access the predictive value for disease risk. The statistical software needed is SPSS version 21 and Medcalc (version 19.1). A value of $p<0.05$ was considered statistically significant.

\section{Ethics approval and consent to participate}

The study was approved by the Ethics Committee of Zhongshan Hospital, Xiamen University and Second affiliated Hospital of Fujian Medical University.

\section{Availability of data and material}

All data generated or analyzed during this study are included in this published article.

\section{AUTHOR CONTRIBUTIONS}

Conception and design: Y-Q Cai, X-B Zhang, and H-Q Zeng. Collection and assembly of data: Y-Q Cai, X-B Zhang, X-J Wei, Z-Y Zhang, L-D Chen, L Hu, Q Ming,
Q-P Peng. Data analysis and interpretation: Y-Q Cai, XB Zhang, and H-Q Zeng. Manuscript writing: All authors. Final approval of manuscript: All authors.

\section{ACKNOWLEDGMENTS}

The authors would like to thank Jihong Liu, dean of Tongji Hospital Affiliated to Tongji Medical College of Huazhong University of science and technology, for the support in data collection.

\section{CONFLICTS OF INTEREST}

The authors declare that they have no conflicts of interest.

\section{FUNDING}

This work was supported by National Natural Science Foundation of China [82170103], the Young people training project from Fujian Province Health Bureau [Grant 2020GGB057] and Xiamen Medical and Health Guidance Project [3502Z20214ZD1043].

\section{REFERENCES}

1. Lai CC, Shih TP, Ko WC, Tang HJ, Hsueh PR. Severe acute respiratory syndrome coronavirus 2 (SARS-CoV2) and coronavirus disease-2019 (COVID-19): The epidemic and the challenges. Int J Antimicrob Agents. 2020; 55:105924.

https://doi.org/10.1016/j.ijantimicag.2020.105924 PMID:32081636

2. Verelst F, Kuylen E, Beutels P. Indications for healthcare surge capacity in European countries facing an exponential increase in coronavirus disease (COVID19) cases, March 2020. Euro Surveill. 2020; 25:2000323.

https://doi.org/10.2807/1560-7917.ES.2020.25.13. 2000323 PMID:32265003

3. Ji Y, Ma Z, Peppelenbosch MP, Pan Q. Potential association between COVID-19 mortality and healthcare resource availability. Lancet Glob Health. 2020; 8:e480. https://doi.org/10.1016/S2214-109X(20)30068-1 PMID:32109372

4. Velavan TP, Meyer CG. Mild versus severe COVID-19: Laboratory markers. Int J Infect Dis. 2020; 95:304-07. https://doi.org/10.1016/j.ijid.2020.04.061 PMID:32344011

5. Yang $X, Y u Y, X u$ J, Shu $H$, Xia J, Liu H, Wu Y, Zhang L, Yu $Z$, Fang $M, Y u T$, Wang $Y$, Pan $S$, et al. Clinical course and outcomes of critically ill patients with SARS-CoV-2 pneumonia in Wuhan, China: a single-centered, 
retrospective, observational study. Lancet Respir Med. 2020; 8:475-81.

https://doi.org/10.1016/S2213-2600(20)30079-5

PMID:32105632

6. Zheng Z, Peng F, Xu B, Zhao J, Liu H, Peng J, Li Q, Jiang C, Zhou Y, Liu S, Ye C, Zhang P, Xing Y, et al. Risk factors of critical \& mortal COVID-19 cases: A systematic literature review and meta-analysis. J Infect. 2020; 81:e16-25.

https://doi.org/10.1016/i.jinf.2020.04.021

PMID: $\underline{32335169}$

7. Colombi D, Bodini FC, Petrini M, Maffi G, Morelli N, Milanese G, Silva M, Sverzellati N, Michieletti E. Wellaerated Lung on Admitting Chest CT to Predict Adverse Outcome in COVID-19 Pneumonia. Radiology. 2020; 296:E86-96.

https://doi.org/10.1148/radiol.2020201433

PMID:32301647

8. Giamarellos-Bourboulis EJ, Netea MG, Rovina N, Akinosoglou K, Antoniadou A, Antonakos N, Damoraki G, Gkavogianni T, Adami ME, Katsaounou P, Ntaganou M, Kyriakopoulou M, Dimopoulos G, et al. Complex Immune Dysregulation in COVID-19 Patients with Severe Respiratory Failure. Cell Host Microbe. 2020; 27:992-1000.e3.

https://doi.org/10.1016/i.chom.2020.04.009

PMID:32320677

9. Qin C, Zhou L, Hu Z, Zhang S, Yang S, Tao Y, Xie C, Ma K, Shang K, Wang W, Tian DS. Dysregulation of Immune Response in Patients With Coronavirus 2019 (COVID19) in Wuhan, China. Clin Infect Dis. 2020; 71:762-68. https://doi.org/10.1093/cid/ciaa248 PMID:32161940

10. Jiang M, Guo Y, Luo Q, Huang Z, Zhao R, Liu S, Le A, Li J, Wan L. T-Cell Subset Counts in Peripheral Blood Can Be Used as Discriminatory Biomarkers for Diagnosis and Severity Prediction of Coronavirus Disease 2019. J Infect Dis. 2020; 222:198-202.

https://doi.org/10.1093/infdis/jiaa252

PMID:32379887

11. Liu J, Li S, Liu J, Liang B, Wang X, Wang H, Li W, Tong Q, Yi J, Zhao L, Xiong L, Guo C, Tian J, et al. Longitudinal characteristics of lymphocyte responses and cytokine profiles in the peripheral blood of SARS-CoV-2 infected patients. EBioMedicine. 2020; 55:102763.

https://doi.org/10.1016/j.ebiom.2020.102763

PMID:32361250

12. Liu Y, Du X, Chen J, Jin Y, Peng L, Wang HH, Luo M, Chen L, Zhao Y. Neutrophil-to-lymphocyte ratio as an independent risk factor for mortality in hospitalized patients with COVID-19. J Infect. 2020; 81:e6-12. https://doi.org/10.1016/j.jinf.2020.04.002 PMID:32283162
13. Liu Y, Yang Y, Zhang C, Huang F, Wang F, Yuan J, Wang Z, Li J, Li J, Feng C, Zhang Z, Wang L, Peng L, et al. Clinical and biochemical indexes from 2019-nCoV infected patients linked to viral loads and lung injury. Sci China Life Sci. 2020; 63:364-74.

https://doi.org/10.1007/s11427-020-1643-8 PMID:32048163

14. Mehta V, Goel S, Kabarriti R, Cole D, Goldfinger M, Acuna-Villaorduna A, Pradhan K, Thota R, Reissman S, Sparano JA, Gartrell BA, Smith RV, Ohri N, et al. Case Fatality Rate of Cancer Patients with COVID-19 in a New York Hospital System. Cancer Discov. 2020; 10:935-41.

https://doi.org/10.1158/2159-8290.CD-20-0516 PMID:32357994

15. Zhang R, Ouyang H, Fu L, Wang S, Han J, Huang K, Jia $M$, Song $Q$, Fu Z. CT features of SARS-CoV-2 pneumonia according to clinical presentation: a retrospective analysis of 120 consecutive patients from Wuhan city. Eur Radiol. 2020; 30:4417-26. https://doi.org/10.1007/s00330-020-06854-1 PMID:32279115

16. Xia X, Wen M, Zhan S, He J, Chen W. [An increased neutrophil/lymphocyte ratio is an early warning signal of severe COVID-19]. Nan Fang Yi Ke Da Xue Xue Bao. 2020; 40:333-36.

https://doi.org/10.12122/j.issn.1673-4254.2020.03.06 PMID:32376581

17. Tang N, Li D, Wang X, Sun Z. Abnormal coagulation parameters are associated with poor prognosis in patients with novel coronavirus pneumonia. J Thromb Haemost. 2020; 18:844-47.

https://doi.org/10.1111/jth.14768 PMID:32073213

18. Terpos E, Ntanasis-Stathopoulos I, Elalamy I, Kastritis E, Sergentanis TN, Politou M, Psaltopoulou T, Gerotziafas G, Dimopoulos MA. Hematological findings and complications of COVID-19. Am J Hematol. 2020; 95:834-47.

https://doi.org/10.1002/ajh.25829 PMID:32282949

19. Zhang L, Yan X, Fan Q, Liu H, Liu X, Liu Z, Zhang Z. Ddimer levels on admission to predict in-hospital mortality in patients with Covid-19. J Thromb Haemost. 2020; 18:1324-29.

https://doi.org/10.1111/jth.14859

PMID:32306492

20. Fu J, Kong J, Wang W, Wu M, Yao L, Wang Z, Jin J, Wu $D, Y u X$. The clinical implication of dynamic neutrophil to lymphocyte ratio and D-dimer in COVID-19: A retrospective study in Suzhou China. Thromb Res. 2020; 192:3-8.

https://doi.org/10.1016/j.thromres.2020.05.006 PMID: $\underline{32407937}$ 
21. Zhang L. Response to "Uncertainties on the prognostic value of D-dimers in COVID-19 patients". J Thromb Haemost. 2020; 18:2067-68. https://doi.org/10.1111/ith.14899 PMID:32407600

22. Li K, Fang Y, Li W, Pan C, Qin P, Zhong Y, Liu X, Huang $M$, Liao $Y$, Li S. CT image visual quantitative evaluation and clinical classification of coronavirus disease (COVID-19). Eur Radiol. 2020; 30:4407-16. https://doi.org/10.1007/s00330-020-06817-6 PMID:32215691

23. Chen N, Zhou M, Dong X, Qu J, Gong F, Han Y, Qiu Y, Wang J, Liu Y, Wei Y, Xia J, Yu T, Zhang X, Zhang L. Epidemiological and clinical characteristics of 99 cases of 2019 novel coronavirus pneumonia in Wuhan, China: a descriptive study. Lancet. 2020; 395:507-13. https://doi.org/10.1016/S0140-6736(20)30211-7 PMID:32007143

24. Li M, Lei P, Zeng B, Li Z, Yu P, Fan B, Wang C, Li Z, Zhou J, Hu S, Liu H. Coronavirus Disease (COVID-19): Spectrum of CT Findings and Temporal Progression of the Disease. Acad Radiol. 2020; 27:603-08. https://doi.org/10.1016/i.acra.2020.03.003 PMID:32204987

25. Liu J, Chen T, Yang H, Cai Y, Yu Q, Chen J, Chen Z, Shang $\mathrm{QL}, \mathrm{Ma} \mathrm{C}$, Chen X, Xiao E. Clinical and radiological changes of hospitalised patients with COVID-19 pneumonia from disease onset to acute exacerbation: a multicentre paired cohort study. Eur Radiol. 2020; 30:5702-08.

https://doi.org/10.1007/s00330-020-06916-4

PMID:32385648

26. Zhang B, Zhang J, Chen H, Chen L, Chen Q, Li M, Chen Z, You J, Yang K, Zhang S. Novel coronavirus disease 2019 (COVID-19): relationship between chest CT scores and laboratory parameters. Eur J Nucl Med Mol Imaging. 2020; 47:2083-89.

https://doi.org/10.1007/s00259-020-04854-3

PMID:32399620

27. National Health and Health Commission of the people's Republic of China. Diagnosis and Treatment of Pneumonia of New Coronavirus Infection. http://www.nhc.gov.cn/yzygi/s7653p/202003/46c929 4a7dfe4cef80dc7f5912eb1989.shtml

28. Chung $M$, Bernheim A, Mei X, Zhang $N$, Huang $M$, Zeng X, Cui J, Xu W, Yang Y, Fayad ZA, Jacobi A, Li K, Li S, Shan H. CT Imaging Features of 2019 Novel Coronavirus (2019-nCoV). Radiology. 2020; 295:202-07. https://doi.org/10.1148/radiol.2020200230 PMID:32017661 